\title{
Adsorption Behavior of the Cetyltrimethylammonium Chloride Reverse Micelles on Porous Silica Gels
}

\author{
Kouta Nakada,* Imdad U. MohammadzaI, ${ }^{* *}$ Satoshi Tsukahara,* and Terufumi Fujiwara*广 \\ *Department of Chemistry, Graduate School of Science, Hiroshima University, \\ Kagamiyama, Higashi-Hiroshima 739-8526, Japan \\ **Department of Chemistry, University of Peshawar, NWFP, Pakistan
}

\begin{abstract}
The adsorption behavior of reverse micelles of cetyltrimethylammonium chloride (CTAC) onto porous silica gels of various pore sizes has been investigated. A decrease in the amount of CTAC in the reversed micellar bulk solution in chloroform/water added to the silica gel in a column was observed, indicating the CTAC adsorption onto the silica surface. The adsorption profile obtained showed a maximum of the adsorbed surfactant amount, where the point of surface saturation may be reached, beyond which the adsorption amount remained unchanged. The adsorption of CTAC was also dependent on the specific surface area of the silica gel used. These results suggest that a CTAC monolayer may be formed and completed on the silica surface. The adsorbed CTAC amount slightly decreased with increasing the water to surfactant molar ratio, $R$, in the reversed micellar bulk solution, although at $R<2$ the CTAC adsorption remained constant with silica gels having larger pore diameters. Even in the presence of traces of water moisture in the bulk solution used for the CTAC adsorption, the adsorption of water on silica gel was also observed and the molar ratios, $R_{\Gamma}$, of the water to surfactant adsorbed on different silica gels were almost unity, implying that the silica gel may tend to hold some of water molecules originally adsorbed on its surface. The adsorption of CTAC molecules appears to occur through the adsorbed water molecules, probably polarized at the silica surface, and a CTAC-water-silica nanointerface seems to be formed. The adsorbed water amount or $R_{\Gamma}$ also increased with an increase in $R$ and on silica gels with larger pore diameters and a plateau was observed in the $R$ range of 3 - 4, beyond which an increase in $R_{\Gamma}$ occurred again. The water molecules present in the vicinity of the adsorbed CTAC and the silica surface may possess different properties at different $R$ and $R_{\Gamma}$ values.
\end{abstract}

(Received August 29, 2008; Accepted October 9, 2008; Published February 10, 2009)

\section{Introduction}

As seen from both industrial and academic viewpoints, the adsorption of ionic surfactants on mineral surfaces is a topic of great interest. The adsorption of surfactants on solid/liquid interfaces is important in several processes, for example, detergency, floatation, wetting behavior, biological phenomena, and pollution control. ${ }^{1}$ Surfactants distribute to an interface and as a result modify the interfacial properties significantly.

Successful attempts were made in the past to stabilize normal micelles on solid supports and the process of immobilization was thoroughly investigated. ${ }^{2-4}$ At interfaces, the self-assembly process is influenced by interactions of several types: surfactantsurfactant, surfactant-surface, surfactant-solvent, and solventsurface. Important results related to the mechanism of adsorption, organization on the solid-liquid interface, concentration dependency, energy considerations, and reproducibility are well documented. The results from these studies are directed towards postulates about the detailed nature of adsorbed ionic surfactants at solid-water interfaces, including reverse hemimicelles, ${ }^{5}$ admicelles ${ }^{6}$ bilayers and multilayers. ${ }^{7}$ It is suggested that the structure of the surfactant adsorbed is, in some respects, comparable to that of the bulk surfactant

$\dagger$ To whom correspondence should be addressed.

E-mail: tfuji@sci.hiroshima-u.ac.jp micelles. ${ }^{5}$ Even though the overall situation at the solid-liquid interface is very complex and can be compared to bulk micellar systems, less is known about the exact structure or shape of the micelles adsorbed at the solid substrate. Although the adsorption of normal micelles from aqueous bulk is well reported, ${ }^{8-13}$ to our knowledge, there appears to be fewer reports concerning the immobilization of conventional reverse micelles on solid supports such as hydrophilic silica gel and mica or hydrophobic graphite, and on their analytical applications.

The potential applications of reversed micellar media and their analytical uses have been reviewed. ${ }^{14,15}$ In the formation of reverse micelles, the hydrocarbon tails are protruding into the bulk apolar solvent while the polar head groups are in contact with the tiny water pool in the core of the reverse micelle. The shape and size of the reverse micelle or the reversed micellar water pool play a significant role in the properties of reversed micellar solutions. The surfactant-water pool nanointerface provides a unique and versatile reaction field and the reversed micelles are referred to as microreactors, ${ }^{16,17}$ which can be exploited for kinetic studies as well as analytical purposes. For example, the use of reversed micellar media in chemiluminescence (CL) analysis has been reported. ${ }^{14,15}$ The sensitivity of the CL reaction can be influenced by changing, for example, the structure of reverse micelles through a change in the value of the water to surfactant molar ratio, $R$ $\left(=\left[\mathrm{H}_{2} \mathrm{O}\right] /[\right.$ surfactant $\left.]\right)$. It is believed that the water to surfactant interface of reverse micelles plays an important role in the 
reversed micellar-mediated CL reaction. ${ }^{18,19}$ Using reverse micelles of cetyltrimethylammonium chloride (CTAC) as a medium in CL analysis, the benefit of CL enhancement was achieved. Also an on-line solvent extraction technique was combined with the reversed micellar-mediated CL detection. ${ }^{20-23}$

There are several reports concerning the application of reversed micellar media as mobile phases in liquid chromatography, ${ }^{24,25}$ TLC, ${ }^{26,27}$ and SFC; $^{28}$ certain problems related to the adsorption of the surfactants were also reported..$^{25}$ The adsorption of cationic surfactants at solid/liquid interface is dependent on the nature of substrate and conditions of the medium, e.g., concentration of the surfactant, $\mathrm{pH}$, ionic strength, and electrostatic as well as hydrophobic interactions are significant in it. ${ }^{29-35}$ Studies related to the environmental applications dealing with the removal of toxic non-ionic organic substances, ${ }^{29,30,32-35}$ and the co-adsorbed harmful metal ions ${ }^{31}$ from sewage materials and soils have been reported. Our interest in the immobilization of reverse micelles stems from the utilities of reversed micellar media mentioned above for analytical purposes. In our preliminary work, reverse micelles of the surfactant CTAC were successfully adsorbed on a glass surface. Since we were encouraged by the findings, this study was extended to the adsorption of CTAC reverse micelles onto porous silica gels. The aims of the present work are to study the adsorption behavior of the CTAC and to explore the function of water that is adsorbed on the silica surface and appears as trapped between the CTAC and silica surface. The overall adsorbed CTAC and water on a silica gel may present a reversed micellar-like situation, where the surfactant may adsorb to the silica gel through the water molecules adsorbed on the silica surface. The resultant CTAC-water-silica nanointerface is expected to possess some new characteristics and can be considered as a nanoreactor. It is also anticipated that the reversed micellar phase immobilized on a silica gel would bear unique properties of material recognition and separation and could be used as a stationary phase in liquid chromatography. Different silica gels as substrates for the adsorption of the cationic CTAC surfactant and water molecules have been used in the present work. Keeping in view the potential analytical applications of CTAC/water on porous silica gels, the effect of the aforementioned molar ratio $R$ extensively investigated is reported here.

\section{Experimental}

\section{Materials}

Silica gels: Silica Gel 60 with a BET surface area of $700 \mathrm{~m}^{2}$ $\mathrm{g}^{-1}$ and a pore size of $6.5 \mathrm{~nm}$, and Silica Gel $60 \mathrm{~N}$ with a BET surface area of $650 \mathrm{~m}^{2} \mathrm{~g}^{-1}$ and a pore size of $6.0 \mathrm{~nm}$, were purchased from Kanto Chemical Co., Inc. (Tokyo, Japan). Other silica gels, Silica Gel 60 with a BET surface area of 470 $\mathrm{m}^{2} \mathrm{~g}^{-1}$ and a pore size of $7.7 \mathrm{~nm}$, Silica Gel 100 with a BET surface area of $320 \mathrm{~m}^{2} \mathrm{~g}^{-1}$ and a pore size of $10.0 \mathrm{~nm}$, and Daisoge ${ }^{\circledR}$ with a BET surface area of $200 \mathrm{~m}^{2} \mathrm{~g}^{-1}$ and a pore size of $20 \mathrm{~nm}$, were obtained from Yamazen Co., Inc. (Osaka, Japan), from Merck Co., Inc. (Darmstadt, Germany) and from Daiso Co., Inc. (Osaka, Japan), respectively. CTAC (98\%) was purchased from Tokyo Kasei Kogyo Co., Ltd. (Tokyo, Japan). Chloroform (HPLC grade, > 99.7\%, stabilized with amylene), cyclohexane (HPLC grade) and ethanol (reagent grade) were purchased from Kanto Chemical Co., Inc. (Japan). All chemicals were used as received. Deionized water, freshly collected from an Advantec Toyo (Tokyo, Japan) Model GSU901 water purification apparatus, was utilized in the preparation of all reversed micellar solutions. All solutions were made fresh before use. The reversed micellar solutions were made as before ${ }^{36}$ by dispersing a certain volume of water in chloroform containing the CTAC surfactant.

\section{Methods}

For each silica gel used, gravimetric studies were carried out to determine moisture contents through a loss on drying in an oven according to the literature. ${ }^{37}$ The silanol numbers were also measured gravimetrically by heating a weighed amount of the silica gel in a furnace at $1000^{\circ} \mathrm{C}$ for $2 \mathrm{~h}$ according to the manner reported. ${ }^{37}$

Moisture measurements described below for the reversed micellar solutions and the solvents before and after passing through silica gels were carried out coulometrically using a Hiranuma Model AQ-3 aquacounter (Hiranuma Co. Ltd., Shizuoka, Japan). Also, the coulometric experiments confirmed that the water contents in the CTAC solutions prepared in chloroform without addition of water were negligibly small $(R=c a$. 0.3).

The amount of CTAC adsorbed onto silica gel was determined through the quantification of the counter chloride ion of CTAC using a Yokogawa (Tokyo, Japan) Model IC-7000 ion chromatographic analyzer. After the adsorbed CTAC surfactants were retrieved from silica gels as mentioned below using ethanol as an eluent, the sample solutions were made for the ion chromatographic analysis by serial dilution of the eluent with deionized water. PTFE tubing of $1.0 \mathrm{~mm}$ i.d. was used throughout the flow system. A $125 \times 4.9 \mathrm{~mm}$ i.d. Yokogawa Excelpack ICS-A44 anion separation column was employed for the ion chromatographic separation at $40^{\circ} \mathrm{C}$, which was coupled to a $30 \times 4.6 \mathrm{~mm}$ i.d. Yokogawa Excelpack ICS-A4G guard column. A $50-\mathrm{mm}^{3}$ loop was used to load the sample solutions using a syringe manually and then each sample solution was inserted in the stream of the $4.0 \mathrm{mM} \mathrm{Na}_{2} \mathrm{CO}_{3}$ and $4.0 \mathrm{mM}$ $\mathrm{NaHCO}_{3}$ carrier driven at the flow rate of $1.0 \mathrm{~cm}^{3} \mathrm{~min}^{-1}$. The eluting chloride ion from the column was monitored by conductometry.

A weighed amount of a silica gel was added to a column containing $50 \mathrm{~cm}^{3}$ of chloroform, while stirring the chloroform gently so that air pockets are not trapped in the column. The extra chloroform was taken out of the column until the silica gel in the column remained covered just with chloroform. Before the addition of the silica gel and after the elution, the moisture contents of the chloroform were determined coulometrically. The reversed micellar solution was then added to the silica gel in the column, passed through it at a flow rate of $2.3 \mathrm{~cm}^{3} \mathrm{~min}^{-1}$, and was recollected in fractions $\left(100\right.$ and $\left.50 \mathrm{~cm}^{3}\right)$. The moisture content of each eluted fraction was also determined coulometrically. For the removal of the adsorbed CTAC surfactant from the silica gel after a rinse of cyclohexane $\left(40 \mathrm{~cm}^{3}\right)$, it was rinsed with ethanol $\left(10 \mathrm{~cm}^{3}\right)$. For the ethanolic eluent collected, the ion chromatographic measurements were performed as mentioned above to determine the residual concentration of CTAC in the eluent, from which the adsorption of CTAC onto the silica gel was estimated. The complete elution of CTAC from the silica gel with ethanol was also confirmed by the following monitoring: After the rinse of ethanol, the silica gel was suspended in water $\left(10 \mathrm{~cm}^{3}\right)$ by sonication for $2 \mathrm{~min}$ and the suspension was permitted to stand for $10 \mathrm{~min}$ to get a clear supernatant. The ion chromatographic measurements made in a similar manner to that described above showed that the amount of the chloride ion in the resultant supernatant solution was negligible, indicating that almost all the adsorbed CTAC molecules were retrieved from the silica gel in the above manner using ethanol. 


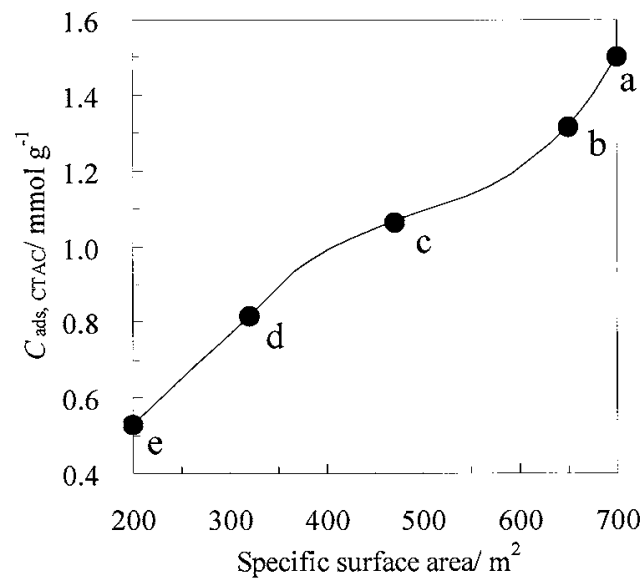

Fig. 1 Variation of the adsorbed CTAC amount $\left(C_{\text {ads,CTAC }}\right)$ with the specific surface area of silica gel. Pore size of silica gel: $a, 6.5 ; b$, $6.0 ; \mathrm{c}, 7.7$;, $10 ; \mathrm{e}, 20 \mathrm{~nm}$.

\section{Results and Discussion}

\section{Adsorption of the CTAC surfactant}

The adsorption of ionic surfactants on a silica surface is greatly affected by the length of their hydrophobic chains ${ }^{30}$ as well as by the ionic strength ${ }^{29}$ and $\mathrm{pH}^{29-35}$ of the aqueous media. The coulombic attractive forces between the ionic surfactant head group and the silica surface covered with water molecules may cause the adsorption of the surfactant molecules and the association of the surfactants to form aggregates on the surface, and the hydrophobic character of the hydrocarbon tail orients away from the surface.

Different volumes of a 0.025 M CTAC solution were passed through $1 \mathrm{~g}$ of the Silica Gel 60 (a BET surface area of $700 \mathrm{~m}^{2}$ $\mathrm{g}^{-1}$ and a pore size of $6.5 \mathrm{~nm}$ ) at a flow rate of $2.3 \mathrm{~cm}^{3} \mathrm{~min}^{-1}$. In the beginning, a rapid increase in adsorption of the surfactant onto the silica gel was observed with increasing the volume of the CTAC solution, probably followed by the formation of surface aggregates. This adsorption process was culminated when $80 \mathrm{~cm}^{3}$ of the $0.025 \mathrm{M}$ CTAC solution were passed through the silica gel and a maximum adsorption amount of 2.1 $\mu \mathrm{mol} \mathrm{m}{ }^{-2}$ CTAC was obtained. With lower flow rates of the CTAC solution, the same adsorption behavior was also observed, suggesting that in the adsorption process an equilibrium state was attained where an exchange of CTAC monomers takes place between the bulk and surface. When CTAC solutions of higher concentrations were used, furthermore, the adsorption profiles remained the same although smaller volumes of the corresponding solutions were needed. At the point of surface saturation, the silica surface may be covered completely with a monolayer of surfactant molecules in a hemimicellar fashion.

The diameter of the pore is one of the important parameters that describe the total area available for adsorption. The CTAC adsorption measurements were also carried out for other silica gels of different pore diameters using the $0.025 \mathrm{M}$ CTAC solution. Figure 1 shows that the adsorption of CTAC increased as the specific surface area of the silica gel increased. On the other hand, the adsorption of CTAC declined with a decrease in the pore size; a maximum amount of the adsorbed CTAC per unit area was obtained on silica gels with relatively larger pore diameters (10 and $20 \mathrm{~nm}$ ). Moreover, the numbers of silanol groups per unit area of the silica surface were estimated for the different silica gels used. With an increase in the size of the

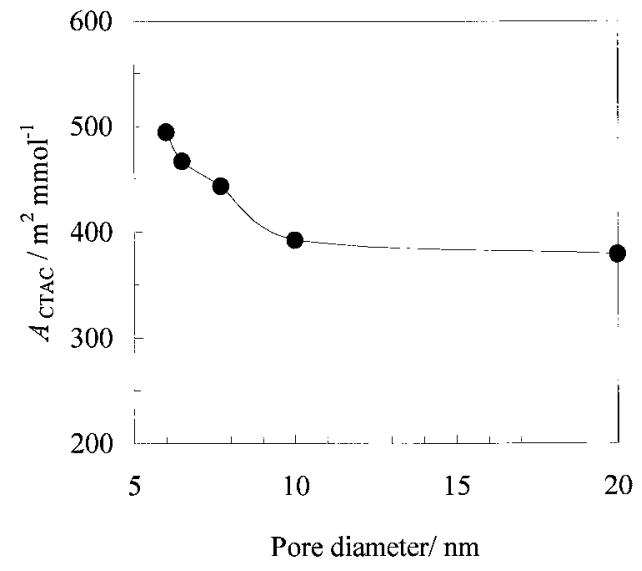

Fig. 2 Variation of the occupation area of the adsorbed CTAC $\left(A_{\text {CTAC }}\right)$ with the pore size of silica gel.

pore diameter, no significant change in the number of silanol groups per unit area was observed, although the adsorption amount of CTAC per unit area increased. These results imply that repulsion between positively charged heads of the surfactant molecules adsorbed on silica gels of smaller pore sizes may be felt and thus seems to limit the CTAC adsorption. In the pores having smaller diameters, it is also likely that steric factors among the bulky CTAC molecules adsorbed cause reduction in the adsorption.

An interesting observation was a relationship between the area occupied by a mole of CTAC adsorbed on the silica surface and the pore diameter, as shown in Fig. 2. With an increase in the pore size, the occupation area per mole of CTAC decreased and remained almost unchanged above a pore diameter of $10 \mathrm{~nm}$. In addition, even when the pore having a larger diameter of $20 \mathrm{~nm}$ was used, the surfactant occupation area obtained was larger than that of $c a .270 \mathrm{~m}^{2} \mathrm{mmol}^{-1}$ at the water to surfactant interface of the reverse micelle in the bulk micellar solution. ${ }^{38}$ These observations provided a strong support to the idea that, in the pores having smaller diameters $(<10 \mathrm{~nm})$, repulsion between the adsorbed surfactant molecules may be enhanced and thus the adsorption seems to reduce.

\section{Adsorption of reverse micelles of CTAC/water on silica gel}

The silica surface is more hydrophilic and is usually covered with water molecules. Also, the polar heads of the surfactant molecules possess a relatively hydrophobic behavior ${ }^{39}$ and may not be in contact with the silica surface directly. The adsorption of water onto the silica surface is a significant parameter that has an impact on the adsorption of the CTAC. Also, the molar ratio $R$ is one of the important factors that affect the physicochemical properties of reverse micelles, since changing the $R$-value simply brings about a modification of the size of the reverse micelle. ${ }^{16,17}$ The water adsorption behavior was thus examined at the different $R$-values of the reversed micellar bulk solutions. The $R$-value was varied by changing the amount of water at a fixed concentration of the CTAC surfactant $([\mathrm{CTAC}]=0.10 \mathrm{M})$ in the reversed micellar bulk solution; thus, the amounts of CTAC available at the surface of the silica gel remained the same. Moisture measurements for chloroform, used as the reversed micellar bulk solvent, confirmed that the water contents in the chloroform before and after passing through silica gels remained almost unchanged. Both the water attached originally to the silica surface and the water of the aqueous pool of the reverse micelle could contribute to the 

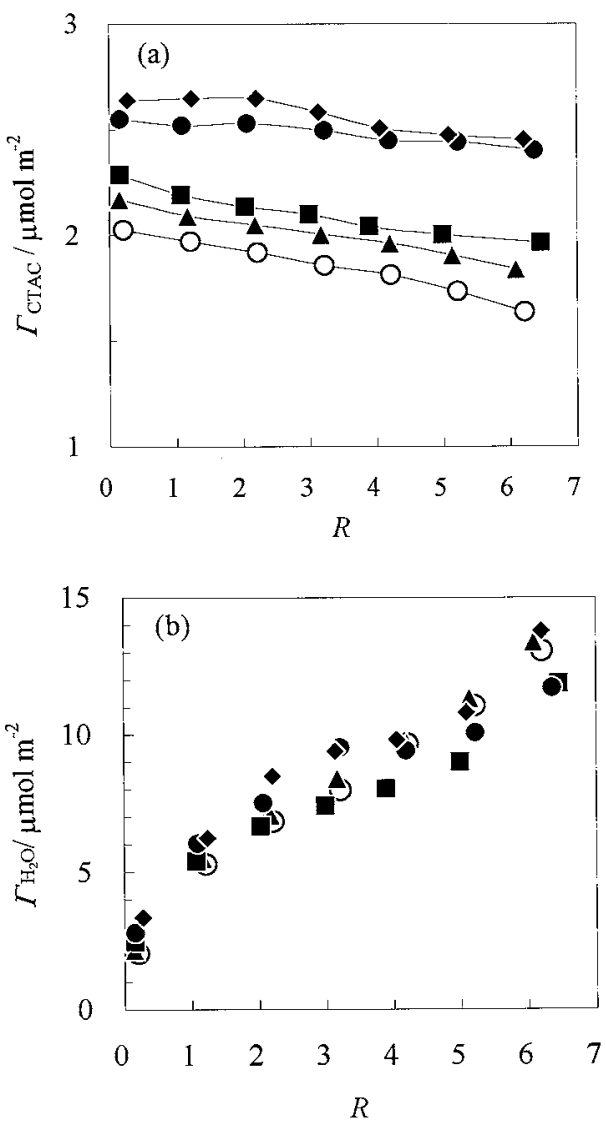

Fig. 3 Effect of $R$ of the reversed micellar bulk solution on the CTAC (a) and water (b) adsorptions onto silica gels with pore diameters of $6.0(0), 6.5(\mathbf{\Delta}), 7.7(\bullet), 10.0(\bullet)$, and $20(\bullet) \mathrm{nm}$.

residual moisture in the eluent. Also, an increase in $R$ could cause an increase in the size of the reversed micellar water pool, thus resulting in an increase in water contribution from the micellar bulk. At lower $R$-values, on the other hand, the amounts of water detected in the eluents were larger than those available originally in the reversed micellar solutions used. This suggests that at the lower $R$ the sizes of the aqueous pools in the micellar bulk are relatively small and may be thus enlarged by picking the water present originally from the silica surface, where the water might be replaced partly with the surfactant molecules.

At varied $R$-values in the reversed micellar bulk solutions, Figs. $3 \mathrm{a}$ and $3 \mathrm{~b}$ show the estimated amounts of surfactant adsorbed $\left(\Gamma_{\text {CTAC }}\right.$ in $\left.\mu \mathrm{mol} \mathrm{m} \mathrm{m}^{-2}\right)$ and of water adsorbed $\left(\Gamma_{\mathrm{H}_{2} \mathrm{O}}\right.$ in $\mu \mathrm{mol} \mathrm{m}{ }^{-2}$ ) onto different silica gels, respectively. Interestingly, with an increase in the $R$-value, that is, with an increase in the size of the aqueous pool of the reverse micelle in the bulk, $\Gamma_{\text {СTAC }}$ showed a mild decline while $\Gamma_{\mathrm{H}_{2} \mathrm{O}}$ enhanced significantly, indicating a preferential adsorption of water over the surfactant. In this case of adsorption of reverse micelles as well as the aforementioned case of the adsorption of CTAC alone, the larger amounts of the adsorbed CTAC per unit area on the silica gels were observed with the wider pore diameters (Fig. 3a). On the other hand, this was not the situation for the adsorption of water, which followed a three-stage isotherm, i.e., $R=0.3-3,3-4$, 4 - 6 (Fig. 3b). Even in the presence of only traces of water moisture in the CTAC solution $(R=c a .0 .3)$ used for the CTAC adsorption, the presence or adsorption of water was observed for silica gels, implying that the silica gel may tend to hold some of

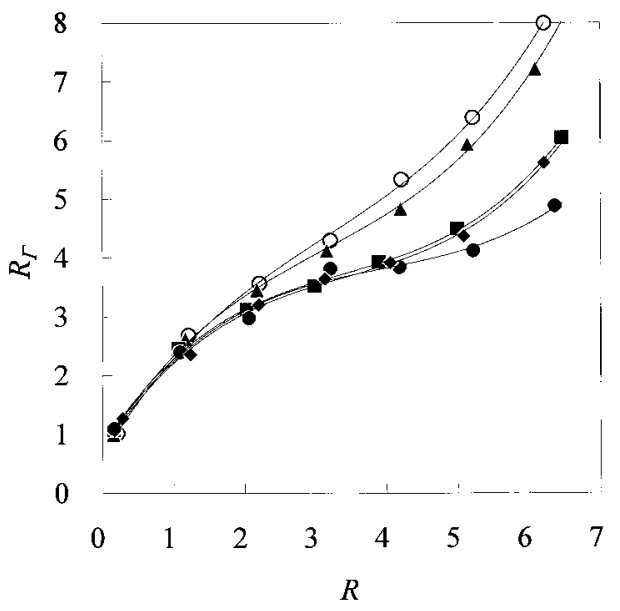

Fig. 4 Effect of $R$ of the reversed micellar bulk solution on $R_{\Gamma}$ for the CTAC reverse micelle adsorbed onto silica gels with pore diameters of $6.0(\bigcirc), 6.5(\boldsymbol{\Delta}), 7.7(\mathbf{\square}), 10.0(\bullet)$, and $20(\bullet) \mathrm{nm}$.

water molecules originally adsorbed on its surface. Up to $R=2$, similar adsorption behaviors of water were observed on all types of silica gels but these changed at higher $R$ values. In this region, there was a sharp increase in the adsorption of water while the CTAC adsorption remained constant with silica gels having larger pore diameters. In the $R$ range of $3-4$, a plateau was obtained for $\Gamma_{\mathrm{H}_{2} \mathrm{O}}$ on silica gels with larger pore diameters. Beyond $R=4$ an increase in $\Gamma_{\mathrm{H}_{2} \mathrm{O}}$ occurred again.

Furthermore, the molar ratios, $R_{\Gamma}\left(=\Gamma_{\mathrm{H}_{2} \mathrm{O}} / \Gamma_{\mathrm{CTAC}}\right)$, of the adsorbed water to the adsorbed CTAC were estimated for various silica gels. The plots of $R_{\Gamma}$ versus $R$ are given in Fig. 4, similar to the curves observed for the adsorption of water at different $R$-values (Fig. 3b). Even when the concentration of water in the bulk solution was reduced to almost zero $(R=c a$. $0.3)$, the $R_{\Gamma}$ ratios for all the silica gels of different pore diameters were observed to be the same and almost unity. This implies that, although loss of the water adsorbed originally on the silica surface may be caused partly as mentioned above, a monolayer of water molecules may be held between the silica surface and the aforementioned monolayer of surfactant molecules, and the water molecules seem to be tightly bound to the surface, likely owing to the cooperative influence of the counter chloride ions of the CTAC surfactants and silanol groups of the surface through hydrogen bonding. For MCM-41 samples having a monolayer of water molecules, a strong hydrogenbonded interaction of the adsorbed water molecules with the surface of silica walls has been observed. ${ }^{40}$ Unlike bulk water, it may have no systematic hydrogen-bonding network. Up to $R=3$, the $R_{\Gamma}$ ratio deviated from unity with a rise in the adsorption of water at the silica surface as the surfactant adsorption remained almost constant. In the range of $R=3-4$, the slope was further decreased or a plateau appeared, and a $R_{\Gamma}$ value of 4 was attained around $R=4$. The more remarkable appearance of such a plateau was observed with silica gels of larger pore sizes, where less repulsion between the adsorbed surfactant molecules is likely as mentioned above. Beyond $R=4$ the slope was increased again. In our previous work, it was suggested that the hydration of the counter chloride ions may contribute to the stabilization of the reversed micellar system; up to $R=4$, the micelle formation was observed to be stabilized by increasing $R$ in the reversed micellar bulk solutions. ${ }^{36}$ Therefore, under the assumption that the situation at the silica surface resembles that of a reverse micelle in the bulk, ${ }^{41}$ the adsorbed water molecules, 
other than those in the water monolayer bound to the silica surface, may be mainly associated with the counter chloride ions, and may be involved in forming the hydrated chloride species in the vicinity of the surfactant interface or between the surfactant molecules. For the gas-phase anionic clusters of $\mathrm{Cl}^{-}\left(\mathrm{H}_{2} \mathrm{O}\right)_{\mathrm{n}}$, evidence of water-water hydrogen-bonding networks was provided in $n=4$ and 5, while for $n=2$ and $3, \mathrm{Cl}^{-}$ion is hydrated without hydrogen bonding between the water molecules. ${ }^{42}$ Thus it seems likely that above $R=4$, such a hydrogen-bonding network may operate, the formation of water clusters or a multilayer water system over the silica surface may start, and the water may be more or less like bulk in characteristics. ${ }^{41}$ It is difficult to describe the structure of the hydrated chloride ions precisely in terms of solvation of the ions and hydrogen bonding among the water molecules adsorbed, and further investigations are needed to clarify it.

\section{Conclusions}

In this work, the adsorption of CTAC and water, that is, the reverse micelle onto porous silica gels was demonstrated. The point of surface saturation observed for the CTAC adsorption suggested the formation of a CTAC monolayer on the silica surface. The adsorption of CTAC is dependent on the specific surface area available and the pore diameter of the silica gel. A change in the $R$-value of the bulk micellar solution used for adsorption brings a small change in the CTAC adsorption while the water adsorption is highly dependent on the $R$-value: Even with the lowest $R$, it was observed that the molar ratios, $R_{\Gamma}$, of the water to surfactant adsorbed on silica gels were almost unity, implying that a monolayer of polarized and rigid water molecules may be present between the silica surface and the CTAC monolayer. Increasing the $R$-value caused an increase in the $R_{\Gamma}$ value of the reverse micelle adsorbed onto the silica surface. The adsorption of water followed a three-stage isotherm, suggesting that several kinds of the water adsorbed onto the silica surface may be present at the CTAC-water-silica nanointerface. The precise number of the adsorbed water layers and the nature of the surfactant aggregates are yet to be confirmed.

\section{Acknowledgements}

This work was partially supported by Grant-in-Aid for Scientific Research No. 16550076 from the Ministry of Education, Science, Sports, and Culture, Japan. One of the authors, I. U. M., also thanks JSPS for awarding a Postdoc Fellowship and the University of Peshawar for granting leave.

\section{References}

1. D. W. Fuerstenau and M. Colic, Colloids Surf., A, 1999, 146, 33.

2. D. B. Hough and H. M. Rendall, in "Adsorption from Solution at Solid/Liquid Interface", ed. C. D. Parfitt and C. H. Rochester, 1983, Chap. 6, Academic Press, New York.

3. M. J. Rosen, "Surfactant and Interfacial Phenomena", 2nd ed., 1989, John Wiley \& Sons, New York.

4. L. K. Koopal, in "Surfactant Science Series, Coagulation and Flocculation: Theory and Applications", ed. B. Dobias, 1993, Vol. 47, Chap. 4, Marcel Dekker, New York, 101.

5. P. Chandar, P. Somasundaran, and N. Turro, J. Colloid
Interface Sci., 1987, 117, 31.

6. J. F. Scamehorn, R. S. Schechter, and W. H. Wade, J. Colloid Interface Sci., 1982, 85, 463.

7. Y. L. Chen, S. Chen, and C. I. Frank, J. Colloid Interface Sci., 1992, 153, 244

8. "Packing and Stationary Phases in Chromatographic Techniques", Chromatographic Science Series, ed. K. K. Unger, 1990, Vol. 47, Marcel Dekker, New York.

9. M. Hanson and K. K. Unger, Trends Anal. Chem., 1992, 11, 368.

10. R. P. W. Scott, "Silica Gel and Bonded Phases, Their Production, Preparation and Use in LC", Separation Science Series, 1993, Vol. 4, John Wiley, Chichester.

11. M. Petro and D. Berek, Chromatographia, 1993, 37, 549.

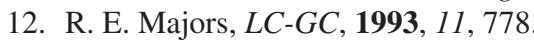

13. B. Buszewski, M. Jezierska, and D. Berek, J. High Resol. Chromatogr., 1998, 21, 267.

14. J. George, Spectrochim. Acta Rev., 1990, 13, 27.

15. W. L. Hinze, in "Organized Assemblies in Chemical Analysis", ed. W. L. Hinze, 1994, Vol. 1, JAI Press, Greenwich, 37.

16. M. P. Pileni, in "Structure and Reactivity in Reverse Micelles", ed. M. P. Pileni, 1989, Elsevier, Amsterdam.

17. M. F. Ruasse, I. B. Blagoeva, R. C. L. Garcia-Rio, J. R. Leis, A. Marques, J. Mejuto, and E. Monnier, Pure Appl. Chem., 1997, 69, 1923.

18. H. Hoshino and W. L. Hinze, Anal. Chem., 1987, 59, 496.

19. T. Fujiwara, I. U. Mohammadzai, K. Kitayama, Y. Funazumi, and T. Kumamaru, J. Colloid Interface Sci., 2007, 310, 682 .

20. T. Kyaw, T. Fujiwara, H. Inoue, Y. Okamoto, and T. Kumamaru, Anal. Sci., 1998, 14, 203.

21. T. Fujiwara, I. U. Mohammadzai, H. Inoue, and T. Kumamaru, Analyst, 2000, 125, 759.

22. T. Fujiwara, I. U. Mohammadzai, K. Murayama, and T. Kumamaru, Anal. Chem., 2000, 72, 1715.

23. T. Fujiwara, I. U. Mohammadzai, H. Inoue, Y. Shimizu, and T. Kumamaru, Anal. Chem., 2003, 75, 4493.

24. M. A. Hernandez-Torres, J. S. Landy, and J. G. Dorsey, Anal. Chem., 1986, 58, 744.

25. A. Berthod, O. Nicolas, and M. Porthault, Anal. Chem., 1990, 62, 1402.

26. D. W. Armstrong and R. Q. Terrill, Anal. Chem., 1979, 51, 2160.

27. A. Mohammad, S. Anwar, and V. Agrawal, J. Indian Soc., 1999, 76, 452 .

28. R. W. Gale, J. L. Fulton, and R. D. Smith, Anal. Chem., 1987, 59, 1977

29. K. Esumi, M. Goino, and Y. Koide, Colloids Surf., A, 1996, $118,161$.

30. P. Favoriti, M. H. Mannebach, and C. Treiner, Langmuir, 1996, $12,4691$.

31. K. Esumi, K. Yoshida, K. Torigoe, and Y. Koide, Colloids Surf., A, 1999, 160, 247.

32. S. Holtzheu, T. Behrend, and R. Herrmann, in "SurfactantBased Separation", ed. J. F. Scamehorn and J. H. Harwell, 2000, Chap. 1, Marcel Dekker, New York, 314.

33. T. Behrend and R. Herrmann, Colloids Surf., A, 2000, 162, 15.

34. D. Talbot, A. Bee, and C. Treiner, J. Colloid Interface Sci., 2003, 258, 20.

35. T. Pradubmook, J. H. O'Haver, P. Malakul, and J. H. Harwell, Colloids Surf., A, 2003, 224, 93.

36. T. Nakashima, T. Fujiwara, and T. Kumamaru, Bull. Chem. Soc. Jpn., 2002, 75, 749. 
37. "Test Method for Silica Gel", Japanese Industrial Standards Committee, Japan (JIS K 1150), 1994, 1.

38. J. Lang, G. Mascolo, R. Zana, and P. L. Luisi, J. Phys. Chem., 1990, 94, 3069.

39. M. W. Rutland and J. L. Parker, Langmuir, 1994, 10, 1110.

40. P. Smirnov, T. Yamaguchi, S. Kittaka, S. Takahara, and Y.
Kuroda, J. Phys. Chem. B, 2000, 104, 5498.

41. T. J. Jain, M. Varshney, and A. Maitra, J. Phys. Chem., 1989, 93, 7409.

42. J. H. Choi, T. K. Kuwata, Y. B. Cao, and M. Okumura, J. Phys. Chem. A, 1998, 102, 503. 